\title{
$\begin{array}{ll}\text { Research Square } & \begin{array}{l}\text { Preprints are preliminary reports that have not undergone peer review. } \\ \text { They should not be considered conclusive, used to inform clinical practice, } \\ \text { or referenced by the media as validated information. }\end{array}\end{array}$ \\ Metabolic Risk Factors in Patients with Kidney Stones with and without Type 2 Diabetes
}

Francisco Spivacow ( $\nabla$ frspivacow@gmail.com )

Universidad del Salvador (USAL)

\section{Elisa Del Valle}

Instituto de Diagnóstico e Investigaciones Metabólicas

Juan Boailchuk

Instituto de Diagnóstico e Investigaciones Metabólicas

\section{Pablo Martínez Allo}

Instituto de Diagnóstico e Investigaciones Metabólicas

\section{Martin Pailler}

Instituto de Diagnóstico e Investigaciones Metabólicas

\section{Research Article}

Keywords: Metabolic Risk Factors, Kidney Stones, Type 2 Diabetes Mellitus, Non-Diabetic Patients

Posted Date: September 22nd, 2021

DOI: https://doi.org/10.21203/rs.3.rs-855238/v1

License: (c) (1) This work is licensed under a Creative Commons Attribution 4.0 International License. Read Full License 
$\underline{\text { RESEARCH ARTICLE }}$

\section{Metabolic risk factors in patients with kidney stones with and without type 2 diabetes}

Spivacow Francisco, ${ }^{1,2}$ del Valle Elisa, ${ }^{1,2}$ Boailchuk Juan, ${ }^{1}$ Martínez Allo Pablo ${ }^{1}$ and Pailler Martín 1

${ }^{1}$ Instituto de Investigaciones Metabólicas (IDIM), ${ }^{2}$ Universidad del Salvador (USAL)

\section{CORRESPONDENCE:}

Francisco R Spivacow

Instituto de Investigaciones Metabólicas

Libertad 836, Piso 1

(C1012AAR) Buenos Aires, Argentina

Phone: +54 11 5031-9700

E-Mail: spiva@idim.com.ar 


\begin{abstract}
Background: in the past few decades, the prevalence of kidney stones in Western countries has increased in parallel with the growing overweight/obesity and type 2 diabetes mellitus rates. An increased insulin resistance in these patients explains, in part, the rising prevalence of uric acid stones. The objective of this retrospective study is to evaluate the metabolic abnormalities in type 2 diabetic and non-diabetic patients with kidney stones. All patients were evaluated following an ambulatory protocol for kidney stones.
\end{abstract}

Methods: A total of 104 diabetic patients (age: $57.8 \pm 11$ years) and 130 non-diabetic (age: 52.1 \pm 6.7 years) patients with kidney stones were selected. Higher rates of body mass index, hypertension, urinary tract infection, gout and hyperuricemia were observed in diabetic patients as compared to the non-diabetics, while similar rates were found for their family history of kidney stones.

Results: Urinary $\mathrm{pH}$ was lower in diabetic patients than in non-diabetic patients. Metabolic abnormalities were detected in $95.2 \%$ and $81.5 \%$ of diabetic and non-diabetic, respectively. Unduly acidic urine $\mathrm{pH}$ was the most frequent abnormality in diabetic patients while hyperuricosuria was the second more common abnormality. On the other hand, in non-diabetics patients idiopathic hypercalciuria was the most common metabolic abnormality followed by hyperuricosuria.

Conclusions: The main risk factor for lithogenesis in type 2 diabetes is unduly acidic urine $\mathrm{pH}$, followed by hyperuricosuria and idiopathic hypercalciuria.

Keywords: Metabolic Risk Factors; Kidney Stones; Type 2 Diabetes Mellitus; Non-Diabetic Patients 


\section{Background}

Kidney stones is currently considered as a systemic condition that is not only limited to the kidney and urinary tract [1] but also it is significantly related to diabetes mellitus (DM), obesity, hypertension (HT), hyperuricemia, hypercholesterolemia and chronic kidney disease (CKD), all of which are well-known cardiovascular risk factors that are usually associated with serios events such as stroke, coronary artery disease or acute myocardial infarction [2,3]. A number of studies have shown an increased prevalence of kidney stones in patients with diabetes mellitus as compared with patients without diabetes [4, 5]. Pathophysiologic explanations for the increased risk of kidney stones in diabetics have largely focused on insulin resistance [6, 7]. Insulin resistance decreases both production and transport of ammonia leading to abnormal urine acidification and low urinary $\mathrm{pH}$ [4]; therefore, this may be expected to favor the production of uric acid (UA) stones since a low urinary $\mathrm{pH}$ is the major lithogenic factor in idiopathic UA nephrolithiasis [8]. On the other hand, the defect in renal acid excretion might also lead to hypocitraturia, an important risk factor for calcium kidney stones [9, 10]. In addition, the compensatory insulin resistance-related hyperinsulinemia may increase urinary calcium excretion $[11,12]$. The objective of this study was to evaluate the metabolic abnormalities in patients with kidney stones and type 2 diabetes mellitus and non-diabetic kidney stone formers.

\section{Methods}

\section{Study design and population}

We conducted a retrospective cross-sectional study in patients with kidney stones and type 2 diabetes mellitus from all over the country from 2009 to 2019 . All patients filled in a form with 
their personal and family medical history. Almost all patients were Caucasian like most of the Argentine population living in large cities. From our database of studies of patients with kidney stones (KS), 104 with type 2 diabetes mellitus and 130 with (KS) without diabetes were selected, matched by sex and age. Since it is a cross-sectional study, we couldn't know with precision the time of evolution of the kidney stone or diabetes in each patient.

\section{Data collection}

The diagnosis of type 2 diabetes was established based on fasting plasma glucose levels $\geq 126$ $\mathrm{mg} / \mathrm{dL}$ or glycated hemoglobin (A1c) levels $\geq 6.5 \%$ or receiving oral antidiabetics or insulin (American Diabetes Association criteria). Among diabetic patients, 81 were taking oral antidiabetics, 20 were receiving insulin, 2 were receiving both oral antidiabetics plus insulin and 1 was undergoing dietary management alone. Kidney stones was diagnosed based on X-ray, ultrasound or computerized tomography (CT) confirmation, or by spontaneous or surgical stone elimination. All patients were evaluated at least one month after the symptomatic kidney stone episode or no longer than 12 months since the last episode; none of them should have ongoing urinary tract infection. Patients with creatinine clearance $(\mathrm{CrCl})$ levels $<60 \mathrm{~mL} / \mathrm{min}$, (corrected for $1.73 \mathrm{~m}^{2} \mathrm{BSA}$ ) were excluded as well as those with prolonged immobilization, or receiving drugs affecting bone metabolism such as corticosteroids, diuretics or anticonvulsants. All patients with kidney stones were evaluated following an ambulatory protocol by which they were asked to continue their usual diet and fluid intake. To reduce bias, two 24-h urine samples were obtained (periods A and B) and kept refrigerated in plastic additive-free containers. Urinary $\mathrm{pH}$ was measured in freshly emitted urine when the patient arrives at the laboratory. If the urinary $\mathrm{pH}(\mathrm{UpH})$ was $<5.5$, a fresh urine $\mathrm{pH}$ determination was repeated and, if persistent, 
a urine saturation test was performed. A fasting blood sample was collected to all patients. The techniques used for the different determinations in blood and urine, were the same as in our previously publication [13]: serum calcium was measured using ion-specific electrode (ISE) testing with a 6 Synchron CX3 automated analyzer (Beckman, Beckman Instruments, Inc. Brea, California, US). The same method was used for urine calcium determination in an acidified aliquot. Serum ionic calcium was measured by ISE testing (Roche Instrument Diagnostic 4 AVL) without $\mathrm{pH}$ correction (normal value $4.5-5.2 \mathrm{mg} / \mathrm{dL}$ ). Both serum and urine creatinine (Jaffe) and phosphate (UV) were measured using an automated analyzer (Spectrum CCX Abbot Labs US). Urine magnesium was measured with Synchron Systems (Calmagite) Reagent using the automated Synchron CX4 analyzer. Both blood and urine sodium and potassium levels were measured with an automated CX3 analyzer. Uric acid levels were measured using the uricase reaction in alkalinized aliquots to prevent precipitation. Urine citrate was determined by an enzyme assay using Sigma-Aldrich Corp. (St. Louis, Missouri, US) reagents. Urine oxalate was measured in an acidified aliquot using an enzyme assay (Trinity Biotech, Co. Bray, Wicklow, Ireland). A $\mathrm{pH}$ electrode was used for measuring the urinary $\mathrm{pH}$ in a fasting urine sample. Cystine determination in urine samples was performed using the Brand chemical reaction. Serum intact parathyroid hormone was measured by IRMA in patients with suspected primary hyperparathyroidism (PHPT). Since our protocol did not include tubular acidification tests, renal tubular acidosis was not included as part of the prespecified diagnostic approach and the cases diagnosed presented during the follow-up period. Idiopathic hypercalciuria (IH) was defined as urinary calcium levels $>300 \mathrm{mg} / 24 \mathrm{~h}$ for men and $>220 \mathrm{mg} / 24 \mathrm{~h}$ for women, or $>4$ $\mathrm{mg} / \mathrm{kg}$ per day for either sex. Hyperuricosuria (HU) was defined as $>800 \mathrm{mg} / 24 \mathrm{~h}$ and $>750$ $\mathrm{mg} / 24 \mathrm{~h}$ for men and women, respectively, or $>600 \mathrm{mg} / \mathrm{L}$ of urine. Hypomagnesuria (HM) was 
defined as $<60 \mathrm{mg} / 24 \mathrm{~h}$, hyperoxaluria (HOx) as $>45 \mathrm{mg} / 24 \mathrm{~h}$, and hypocitraturia (HC) as $<350$ $\mathrm{mg} / 24 \mathrm{~h}$. Unduly acidic urine (UAU) was defined when urinary $\mathrm{pH}$ was $<5.5$ in at least two different measurements on the same day. Cystinuria (CYS) was defined as values $>250 \mathrm{mg} / 24$ h. Hyperuricemia was defined as $>6.5 \mathrm{mg} / \mathrm{dL}$ in women and $>7 \mathrm{mg} / \mathrm{dL}$ in men. Low urine volume (LUV) was diagnosed with values $<1000 \mathrm{~mL} / 24 \mathrm{~h}$. Patients with no established biochemical disorders in their urine which might justify the occurrence of kidney stone were classified as "not metabolically abnormal" (NMA). In all patients with two UpH values $<5.5$, urine saturation was calculated using a computer program (ACTILIT) that assesses the relative risk (RR) of crystallization for different salts. Since other more accurate biochemical techniques are not available at our Institution, of the 104 stone formers with diabetes mellitus, stone composition was determined in $33(32 \%)$ of the patients using a qualitative chemical method (colorimetric, visual comparison) and/or optical crystallography with polarized light and in 27 (21\%) non-diabetic patients with kidney stones.

\section{Informed Consent and Ethical Review}

All the subjects signed an Informed Consent Form. The Informed consent form and the protocol were reviewed and approved by the Institutional Review Board of the Instituto de Investigaciones Metabolicas

\section{Statistical analysis}

The statistical analysis (Student's T-test, Chi-squared test, proportionality and Pearson test) were performed using the CSS program Statisca Software (StatSoft Inc., Tulsa, OK, US). A

$\mathrm{p}>0.5$ was considered statistically significant 


\section{Results}

Table 1 shows the demographic characteristics of both diabetic and non-diabetic patients with kidney stones. Predominance was observed in male patients with diabetes mellitus, with a male: female ratio of 1.4:1. Although height measurements were similar, both weight and body mass index (BMI) were higher in diabetic patients. In patients with type 2 diabetes, hypertension, urinary tract infection, gout or hyperuricemia, and a first- or second-degree family history of kidney stones were observed in $61 \%, 40.2 \%, 18.4 \%$, and $25.5 \%$ of the cases, respectively; while in non-diabetic patients, these were observed in $33 \%, 22.2 \%, 12.5 \%$, and $25 \%$, respectively. Of 33 kidney stones that were analyzed in type 2 diabetes, $19(58 \%)$ corresponded to calcium oxalate, $9(27 \%)$ to uric acid, and $5(15 \%)$ to mixed kidney stones (calcium oxalate/uric acid). In the 27 kidney stones analyzed in patients with non-diabetic kidney stones, $5(18.5 \%)$ were uric acid and $22(81.5 \%)$ were calcium salts (oxalate, phosphate). Urinary pH was lower in type 2 diabetes patients than in non-diabetics $(5.4 \pm 04$ vs. $6.0 \pm 0.4, \mathrm{p}<0.001)$. This difference grew with increasing BMI in type 2 diabetes patients and remained unchanged in non-diabetics. Urinary volume, urine calcium and urine uric acid were comparable between both groups. However, a non-statistically significant slight increase in natriuria was observed in patients with type 2 diabetes as compared to the non-diabetics. Metabolic abnormalities were found in $95.2 \%$ of diabetic patients and in $81.5 \%$ of the non-diabetics. No metabolic abnormalities (NMA patients) were found in $4.8 \%$ of type 2 diabetes patients and in $18.5 \%$ of the non-diabetics. Single metabolic abnormalities were observed in $47.5 \%(n=47)$ of diabetic patients and in $77 \%$ $(n=82)$ of the non-diabetics. Table 2 shows the single metabolic abnormalities in both groups. Single UAU and single HU were more commonly found in diabetic patients; in turn, single hypercalciuria and HU were more common in the non-diabetics. Combined abnormalities in 
both populations are also shown in Table 3, with diabetic patients showing the largest number of combinations. As shown in Figure 1, when analyzing the total (both single and combined) metabolic abnormalities, idiopathic hypercalciuria, hyperuricosuria, hypocitraturia and UAU were more common in the non-diabetics. In type 2 diabetes patients, although IH was the most common abnormality, the values were lower than those in non-diabetic patients. Besides IH, the most important differences included the greater proportion of UAU and the lower occurrence of hypocitraturia in diabetic patients as compared to the non-diabetics. Low urinary volumes were observed in $7 \%$ of the non-diabetics and in $3.9 \%$ of type 2 diabetes patients.

\section{Discussion}

The prevalence of kidney stones in patients with diabetes mellitus is greater than in the overall population [4]. In that sense, Meydan et al [4] have described kidney stones in $21 \%$ of 286 diabetic patients versus $8 \%$ in 111 non-diabetic individuals, almost 3-fold higher. A prospective study by Taylor et al [3] that included 289,900 individuals (men and women of different ages) found a higher risk of kidney stones in diabetic patients up to $68 \%$ in young women (25-43 years of age), $38 \%$ in older women (30-55 years) and $31 \%$ in men, compared to non-diabetic individuals, matched for age and sex. In the other hand, a series by Daudon et al [12] with 2464 patients with kidney stones in Paris found $11 \%$ of cases in diabetic patients, which is higher than the $7.2 \%$ observed by our group in 252 diabetic patients with kidney stones (unpublished data). In our series, the male:female ratio was of $1.4: 1$, similar to the $1.6: 1$ ratio described by Weinberg et al [14], and lower than the 2.2:1 ratio described in 462 diabetic patients with kidney stones in another study [15]. Higher BMI, HT and a history of urinary tract infections were more commonly found in diabetic patients than in non-diabetics. The presence of first or 
second-degree family history of kidney stones was the same in both groups and similar to the one described in a previous series of us in non-diabetic patients with nephrolitiasis [16]. In relation to the composition of kidney stones in diabetic patients, Pak et al [6] found uric acid stones in $34 \%$ of the cases, unlike the $6.2 \%$ in non-diabetic individuals with kidney stones. This data is consistent with the analysis of 2464 patients with uric acid kidney stones, $35.7 \%$ of whom were diabetic and $11 \%$ were non-diabetic [14]. In our series, we were only able to analyze 33 stones type 2 diabetes, and 27 without diabetes. Uric acid stones predominated in patients with diabetes than in non-diabetics, $27 \%$ vs $18.5 \%$. Although this percentage is lower than that previously described, it is higher than the $16.5 \%$ found after analyzing 8885 stones from non-diabetic patients in Argentina [17]. With regards to urinary $\mathrm{pH}$, diabetic patients had lower UpH than non-diabetic patients, and UpH was significantly lower with increasing BMI. This data is consistent with that found by Maalouf et al [18] in 4883 patients with kidney stones who showed inverse relationship between $\mathrm{UpH}$ and body weight. Reduced $\mathrm{UpH}$, characteristic of overweight, obesity and diabetes mellitus $[3,18]$ has been associated with insulin resistance [20-22]. The excessive urine acidity of uric acid stone formers is associated with increased net acid excretion and reduced excretion of ammonia, which normally acts as a buffer for urine acidity [23]. Some researchers have suggested defects in enzymes that metabolize glutamine into ammonia and $\alpha$-ketoglutarate, such as glutaminase and/or glutamate dehydrogenase [24, 25]. Although an acid $\mathrm{UpH}$ is the central cause of uric kidney stone formation in diabetic patients, also lower citrate excretion linked to tubular defects [9] and hypercalciuria associated with compensatory hyperinsulinemia due to insulin resistance [10], would explain the formation of calcium salts stones. Our study found no differences in uric acid and calcium excretion or low urinary volume, but increased natriuresis with respect to the non-diabetics was observed. 
Metabolic abnormalities were detected in $95.2 \%$ and $81.5 \%$ of diabetic and non-diabetic patients, respectively.

Single metabolic abnormalities were observed in $47.5 \%$ and $77 \%$ of diabetic and non-diabetic patients. The most frequent, single metabolic abnormality in diabetic patients was unduly acid urine, while idiopathic hypercalciuria was the most common metabolic abnormality in nondiabetic patients. Hyperuricosuria was the second more common abnormality found in both groups of patients. While hypocitraturia was less common in diabetic patients, hypomagnesuria was similar between the two groups. No cases of hyperoxaluria or cystinuria were found in the diabetic population.

Combined abnormalities were more common (52.5\%) in diabetic than non-diabetic patients $(23 \%)$.

Limitations of the study: not being able to obtain a greater number of kidney stones to analyze in both diabetic and non-diabetic patients. Also, in our institution there are no more current techniques for the analysis of kidney stones.

\section{Conclusions}

As a single alteration, UAU it is the main metabolic diagnosis in type 2 diabetes with kidney stones, unlike idiopathic hypercalciuria in non-diabetic KS patients. This favors the greater presence of uric acid stones given in patients with diabetes. The other two alterations most frequently found in lithiasic diabetic patients are hyperuricosuria and idiopathic hypercalciuria. While in non-diabetics idiopathic hypercalciuria, hyperuricosuria and hypocitraturia are the most common diagnoses. Kidney stones in patients with type 2 diabetes predominate in males, 
and are more commonly associated with hypertension, urinary tract infection, gout and hyperuricemia. Biochemical alterations are diagnosed a few years later than non-diabetic patients and are characterized by being older and having greater BMI thant non-diabetic patients.

\section{Abbreviations}

HT: hypertention; CKD: chronic kidney disease; UA: uric acid; KS: kidney stones; A1c: glycated hemoglobin; CT: computerized tomography; $\mathrm{CrCl}$ : creatinine clearance; $\mathrm{UpH}$ : urinary pH; PHPT: primary hyperparathyroidism; IH: idiopathic hypercalciuria; HU: hyperuricosuria; HM: hypomagnesuria; HOx: hyperoxaluria; HC: hypocitraturia; UAU: unduly acidic urina: CYS: cystinuria; LUV: low urine volume; NMA: not metabolically abnormal; RR: relative risk; BMI: body mass index;

\section{Acknowledgments}

Not applicable.

\section{Authors' contributions}

Study concept and design: S. F and DV. E, Acquisition of data: MA.P and P.M

Data analysis: S.F and DV.E. Review the results and interpreted the data: S.F and DV.E. Writing the manuscript: S.F. Review and revised the manuscript:

DV.E. Translation: B.J. All authors have read and approved the manuscript.

\section{Availability of data and materials}

The datasets used and analysed during the current study available from the 
corresponding author on reasonable request.

\section{Declarations}

\section{Ethics approval and consent to participate}

The methods and regulations in this study were made according to the declaration of Helsinski

\section{Funding}

Not applicable.

\section{Competing interests}

The authors have nothing to disclose.

\section{Author details}

${ }^{1}$ Instituto de Investigaciones Metabólicas (IDIM), Buenos Aires, Argentina. ${ }^{2}$ Universidad del Salvador (USAL), Buenos Aires, Argentina. 


\section{References}

1. Yanqiong Liu SL, Zhiyu Zeng, Jian Wang, Li Xie, Taijie Li et al. Kidney Stones and Cardiovascular Risk: A Meta-analysis of cohort Studies. Am J Kidney Dis. 2014;64(3):402-10.

2. Seolhye K YC, Eunju S, Jeong Gyu K, Kyung Eun Yun, Hyun-Suk Jung et al. Association between sonographically diagnosed neprolithiasis and subclinical coronary rtery calcification in adults. AJKD. 2017;71(1):35-41.

3. Taylor EN, Stampfer MJ, Curhan GC. Diabetes mellitus and the risk of nephrolithiasis. Kidney Int. 2005;68:1230-5.

4. Meydan N, Barutca S, Caliskan S, Camsari T. Urinary stone disease in diabetes mellitus. Scand J Urol Nephrol. 2003;37:64-70.

5. Abate N, Chandalia M, Cabo-Chan Av Jr, Moe OW, Sakhaee K. The metabolic syndrome and uric acid nephrolithiasis: Novel features of renal manifestation of insulin resistance. Kidney Int. 2004;65:386-92.

6. Pak CY, Sakhaee K, Moe O, Preminger GM, Poindexter JR, Peterson RD et al. Biochemical profile of stone-forming patients with diabetes mellitus. Urology. 2003; $61: 523-7$.

7. Pak CY, Poindexter JR, Peterson RD Koska J, Sakhaee K. Biochemical distinction between hyperuricosuric calcium urolithiasis and gouty diathesis. Urology. 2002;60:789-94.

8. Coe FL, Parks JH, Asplin JR. The pathogenesis and treatment of kidney stones. N Engl J Med. 1992;327:1141-52.

9. Hamm LL. Renal handling of citrate. Kidney Int. 1990;38:728-35. 
10. Shimamoto K, Higashiura K, Nakagawa M, Masuda A, Shiiki M, Miyazaki Y et al. Effects of hyperinsulinemia under the euglycemic condition on calcium and phosphate metabolism in non-obese normotensive subjects. Tohoku J Exp Med. 1995;177:271-8.

11. Nowicki M, Kokot F, Surdacki A. The influence of hyperinsulinaemia on calciumphosphate metabolism in renal failure. Nephrol Dial Transplant. 1998;13:2566-71.

12. Daudon M, Traxer O, Conort P, Lacour Bernard, Jungers Paul. Type 2 diabetes Increases the Risk for Uric Acid Stones. J Am Soc Nephrol. 2006;17:2026-33.

13. Spivacow FR, del Valle EE, Negri AL, Fradinger E, Abib A, Rey P.Biochemical diagnosis in 3040 kidney Stone formers in Argentina. Urolithiasis. 2015;43:323-330.

14. Weinberg AE, Patel CJ, Chertow GM, Leppert JT. Diabetic Severity and Risk of Kidney Stone Disease. Eur Urol. 2014;65:242-7.

15. Fang-Yeh Chu, Chih-Chun Chang, Pin-Hao Huang, Yi-Ning Lin, Po-Wen Ku, Jen-Tang Sun, et al. The Association of Uric Acid Calculi with Obesity, Prediabetes, Type 2 Diabetes Mellitus, and Hypertension. Biomed Res Int. 2017;7523960.

16. Spivacow FR, Abdala R, del Valle EE, Loachamin F, Silveira F, Rey P. Family history and biochemical diagnosis in 1948 kidney stone formers. Rev Nefrol Dial Traspl. 2017; $37: 198-206$.

17. Spivacow FR, del Valle EE, Lores E, Rey Paula. Kidney Stones: composition, frequency and relation to metabolic diagnosis. Medicina. 2016:76:343-8.

18. Maalouf NM, Sakhaee K, Parks JH, Coe FL, Adams-Huet B, Pak CY. Association of urinary pH with body weight in nephrolithiasis. Kidney Int. 2004;65(4):1422-5.

19. Trinchieri A, Croppi E, Montanari E. Obesity and urolithiasis: evidence of regional influences. Urolithiasis. 2017;45:271-8. 
20. Pak CY, Sakhaee K, Peterson RD, Poindexter JR, Frawley WH. Biochemical profile of idiopathic uric acid nephrolithiasis Kidney Int. 2001;60:757-61.

21. Eckel RH, Grundy SM, Zimmet PZ. The metabolic syndrome. Lancet. 2005;365:1415-28.

22. Beck-Nielsen H, Groop LC. Metabolic and genetic characterization of prediabetic states. Sequence of events leading to non-insulin-dependent diabetes mellitus. J Clin Invest. 1994; 94:1714-21.

23. Kamel KS, Cheema-Dhadli S, Halperin ML. Studies on the pathophysiology of the low urine pH in patients with uric acid stones. Kidney Int. 2002;61:988-94.

24. Pollak V, Mattenheimer H. Glutaminase activity in the kidney in gout. J Lab Clin Med. 1965;66:564-70.

25. Gutman A, Yue TF. An abnormality of glutamine metabolism in primary gout. Am J Med. $1963 ; 35: 820-31$. 
Page $\mathbf{1 6}$ of $\mathbf{1 6}$ 
Figures

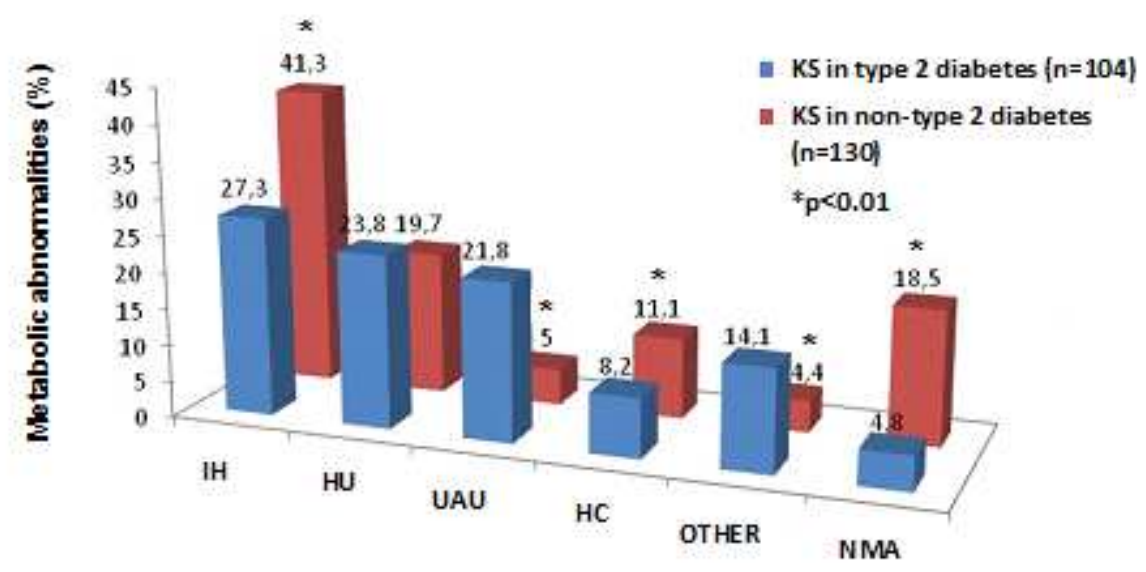

Figure 1

Total (single and combined) metabolic abnormalities in both controls and diabetic patients with kidney stones

\section{Supplementary Files}

This is a list of supplementary files associated with this preprint. Click to download.

- DIABETESANDKIDNEYLITHIASISTABLES.pdf 